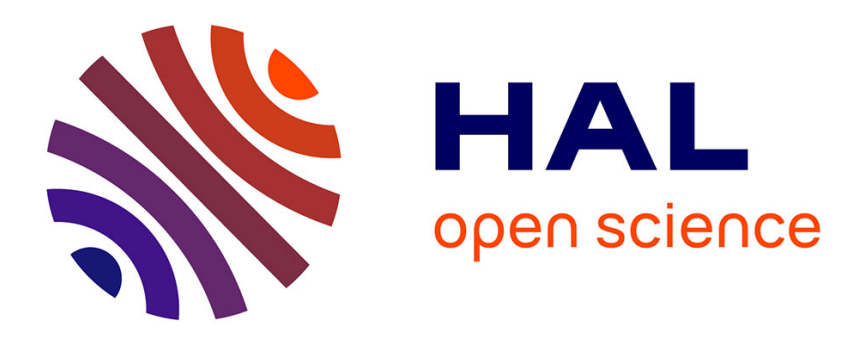

\title{
Unité ouvriers-étudiants : quelles pratiques derrière le mot d'ordre ?
}

\author{
Nicolas Hatzfeld, Cédric Lomba
}

\section{To cite this version:}

Nicolas Hatzfeld, Cédric Lomba. Unité ouvriers-étudiants : quelles pratiques derrière le mot d'ordre ?: Retour sur Besançon en 1968. Savoir / Agir , 2008, Mai-Juin 68: la rencontre ouvriers/étudiants, 6, pp.41-48. 10.3917/sava.006.0041 . hal-01293780

\section{HAL Id: hal-01293780 \\ https://hal.science/hal-01293780}

Submitted on 25 Mar 2016

HAL is a multi-disciplinary open access archive for the deposit and dissemination of scientific research documents, whether they are published or not. The documents may come from teaching and research institutions in France or abroad, or from public or private research centers.
L'archive ouverte pluridisciplinaire HAL, est destinée au dépôt et à la diffusion de documents scientifiques de niveau recherche, publiés ou non, émanant des établissements d'enseignement et de recherche français ou étrangers, des laboratoires publics ou privés. 


\title{
Unité ouvriers-étudiants : quelles pratiques derrière le mot d'ordre ? Retour sur Besançon en 1968
}

\author{
Nicolas Hatzfeld et Cédric Lomba
}

Parmi les questions vives posées dans le cours du mouvement de mai-juin 68 et laissées en héritage, figure en bonne place celle des relations entre étudiants et ouvriers. Des films d'alors témoignent des débats sur ce sujet dans les amphis, d'autres montrent des manifestants étudiants venus du Quartier latin jusqu'aux usines Citroën ou Renault. Ces images montrent un contact sans jonction et alimentent un récit qui présente les deux mouvements - étudiant et ouvrier - comme deux dynamiques parallèles. Ce récit cloisonné a longtemps dominé dans l'historiographie, construite sur la thèse de trois crises : crise étudiante, crise sociale et crise politique. Des travaux récents ${ }^{1}$ contestent cette lecture, insistant sur les «transversalités» et les «métissages» entre ces univers dans de nombreuses villes de France ${ }^{2}$. Nous voudrions aborder cette question de la rencontre à partir du cas de Besançon. Dans cette ville, en effet, différentes traces font état de ces contacts et permettent de s'interroger tant sur leur réalité et leur consistance, que sur les façons dont ils ont été mis en perspective.

\section{À chaud, tableau d'un désaccord parfait}

Outre la figure célèbre en 68 de l'usine de textiles artificiels Rhodiaceta (3 000 personnes), occupée du 17 mai au 8 juin, la ville de Besançon compte de nombreuses usines aux activités aussi diverses que la métallurgie lourde, l'horlogerie ou l'alimentation. Ouvrières et ouvriers sont en grand nombre dans cette ville qui compte, par ailleurs, une université ancienne et active. De ce fait, Besançon est un cas que l'on se gardera de tenir pour exemplaire, mais néanmoins riche de significations sur la manière dont ont pu se tisser

\footnotetext{
${ }^{1}$ Dominique Damamme et alii (dir.), Mai-juin 68, Paris, L'Atelier, 2008 ; Philippe Artières et Michèle ZancariniFournel (dir.), 68, une histoire collective, Paris, La Découverte, 2008 ; Xavier Vigna, L'insubordination ouvrière dans les années 68, Rennes, Presses universitaires de Rennes, 2007.

${ }^{2}$ Boris Gobille, Mai 68, Paris, La Découverte, Repères, 2008, p. 54-57.
} 
des relations entre deux groupes sociaux en présence durant mai-juin 1968.

Dans le cours même des événements, le jour du discours décisif du Général, un envoyé spécial du Nouvel Observateur dresse un tableau ambivalent de la situation : «Le bruit en courait à Paris : la faculté des lettres de Besançon était "enragée". (...) On parlait aussi d'une fraternisation généreuse entre les ouvriers et les étudiants, d'une vraie révolution culturelle - à Rhodiaceta entre autres. (...) À Besançon plus qu'ailleurs, plus profondément qu'ailleurs, il y a eu contact entre les facultés et les usines: mais ce contact a été négatif, la force des combattants n'en a pas été multipliée, comme on l'avait espéré si fort quand la révolution semblait une affaire faite. La rencontre a tout de même eu lieu, entre des gens qui ne se connaissaient pas plus que de Bételgeuse à Alpha du Centaure. De ce que je raconte, des réticences ouvrières (pas toujours "inspirées") et de l'aventurisme livresque des étudiants, du choc confus entre deux subjectivités de classe, qu'on ne déduise pas le désespoir d'impossibles retrouvailles ${ }^{3} \gg$.

Message d'espoir, certes, mais tableau d'un désaccord parfait. L'article développe les composantes de cette discordance entre étudiants et ouvriers. Les premiers viennent de tenter de faire venir les seconds à l'université, en vain. Dans plusieurs usines - métallurgie masculine, confection féminine -, des grévistes leur ont signifié la volonté ouvrière de défendre seuls leurs revendications, limitées à un cadre syndical. Parmi eux, l'emprise du Parti Communiste est présentée comme décisive, au point de créer autour de la Rhodia un cordon sanitaire et de transformer l'usine en forteresse. Quant aux étudiants, présentés avec bienveillance, ils donnent l'image d'un collectif d'avant-garde, habité par un projet de révolution qu'il n'arrive pas à faire adopter par les interlocuteurs ouvriers. Pour évaluer la pertinence de ce tableau, il faut le croiser avec d'autres sources.

\section{Des expériences antérieures}

S'il est extérieur au tissu social de Besançon, Jean-Francis Held, ancien communiste habitué au reportage, a une grande acuité politique. D'autres journalistes ${ }^{4}$, s'en tenant à l'existence des contacts, décrivent une relation plus positive, mais peut-être plus

\footnotetext{
3 Jean-Francis Held, « L'expérience du dialogue », Le Nouvel Observateur, 7 juin 1968.

${ }^{4}$ Le Monde, 20 mai 1968, cité par Nadège Faivre, Mai 68 à la Faculté des Lettres de Besançon, Mémoire d'histoire, Université de Franche-Comté, 1996.
} 
superficielle. La consultation d'autres sources invite à nuancer de deux façons l'image d'un «contact négatif». Tout d'abord, ce n'est pas la première fois qu'ouvriers et étudiants entrent en relations. En 1964, par exemple, un tract de la CFTC remercie une trentaine d'étudiants, employés par la Rhodia pour un emploi d'été, d'avoir refusé de remplacer les ouvriers lock-outés ${ }^{5}$. L'épisode, au passage, montre des étudiants bien différents, dans leur mode de vie, du stéréotype de la « jeunesse dorée ».

C'est surtout durant le conflit avec occupation de la même usine, en février-mars $1967^{6}$, au retentissement national, que les contacts sont les plus intenses. Des militants syndicaux viennent à plusieurs reprises expliquer la situation de la grève à la fac de Lettres devant un amphi d'étudiants. L'UNEF est partie prenante du comité de soutien, distribue des tracts en ville, participe aux manifestations de soutien et organise des quêtes pour la caisse de grève. Plus encore, un groupe d'une trentaine d'étudiants vient régulièrement à l'usine. Un leader ouvrier, militant CFDT devenu cégétiste en 68, raconte aujourd'hui que dès le début de l'occupation, les plus politisés des étudiants viennent immédiatement proposer leur aide : «Les étudiants viennent au piquet, ils sont tout contents : "Qu'est-ce qu'il faut faire?" On les fait bosser (rires). C'est assez sympa... La CGT freine plutôt, la CFDT non, et ils viennent. Le restaurant est ouvert le soir, ils viennent au spectacle. Pas des centaines et des centaines. Mais dans les manifs, ils ramassaient beaucoup de monde. ${ }^{7}$ "Il y a donc deux cercles de soutien étudiant aux ouvriers grévistes, les manifestants occasionnels et les assidus de l'occupation. Ces derniers sont surtout des militants trotskystes. Un ancien étudiant de l'Union des étudiants communistes considère, quant à lui, que les étudiants communistes étaient «peut-être moins fréquemment présents à la Rhodia, en tant que militants comme ça, parce que le lien avec les communistes était constant, et qu'il y avait des communistes dans l'usine. Les gens de la JCR y allaient plus, parce qu'ils n'avaient pas un grand parti ouvrier, impliqué à l'intérieur ${ }^{8} \gg$.

Lorsqu'arrive 68, des étudiants et des ouvriers se sont donc déjà fréquentés et ont plus ou moins appris à prendre la mesure de leurs différences sociales et d'orientation politique.

\footnotetext{
${ }^{5}$ Le Comtois, 10/07/64 ; Tract CGT-CFTC Rhodia, juin 1964, AD Franche-Comté.

${ }^{6}$ Nicolas Hatzfeld, Cédric Lomba, « La grève de la Rhodiaceta », in Dominique Damamme et alii (dir.), op. cit., p. 207-221.

${ }^{7}$ Georges Maurivard, entretien avec N. Hatzfeld et C. Lomba, décembre 2006.

${ }^{8}$ Michel Pagani, entretien avec N. Hatzfeld, décembre 2006.
} 
Ce mode de relations est bousculé en mai-juin.

\section{" Ouvriers-étudiants " contre " mouvement ouvrier-gens de culture "?}

À l'occasion du mouvement, les contacts entre étudiants et ouvriers de la Rhodia sont réactivés ${ }^{9}$. D'emblée, ils s'avèrent plus complexes qu'auparavant. Le lieu de la contestation change. Si l'usine Rhodia est quasiment fermée aux étudiants, c'est d'abord parce qu'elle est désertée par ses ouvriers qui s’impliquent dans le mouvement général. Le PC, au demeurant, serait bien en peine d'imposer sa volonté dans cette usine où domine une CFDT qui mise sur sa combativité pour maintenir son hégémonie. Laissant sur place une permanence, les activistes de la grève élargissent leur territoire d'action à d'autres usines plus petites et non syndiquées qu'ils contribuent à faire débrayer et à l'université. Répondant aux groupes d'étudiants venus aux portes de l'usine, des syndicalistes, surtout CFDT, viennent, plus ou moins régulièrement, aux AG de la fac de Lettres. Selon les souvenirs d'aujourd'hui, ils ont des échanges sur la situation de la classe ouvrière, sur les mobilisations ou sur des questions d'organisation sociale et industrielle, en particulier autour de l'autogestion. L'un d'eux mentionne aussi la découverte joyeuse d'un monde où «l'on s'éclatait dans tous les sens » entre jeunes ${ }^{10}$.

La connivence dépasse parfois les positions officielles des appareils nationaux. C'est ainsi qu'une trentaine d'OS cégétistes de la Rhodia, pour certains membres du PC, regroupés dans l'équipe des « durs », réagissent négativement au « constat » de Grenelle. Accompagnés d'étudiants, ils forment des piquets de grève qui bloquent certaines usines de Besançon et même d'une petite ville voisine ${ }^{11}$. Ces mobilisations communes sont autant d'expériences partagées qui nuancent les représentations clivées de deux mondes en présence. Mais certaines tentatives étudiantes sont à la fois moins consensuelles et moins fructueuses. C'est le cas de contacts pris entre des étudiants de la JCR et quelques jeunes OS de la Rhodia, « révoltés » d'origine rurale et qui avaient été déçus par la reprise du travail en mars 1967 : l'esquisse d'un noyau contestataire ne se concrétise pas ${ }^{12}$.

\footnotetext{
${ }^{9}$ Des ouvriers de la Rhodia prêtent main forte aux étudiants dans un rassemblement devant la fac en septembre 1967.

${ }^{10}$ Nicolas Bultot, entretien avec N. Hatzfeld et C. Lomba, octobre 2007.

${ }^{11}$ id.

${ }^{12}$ Claudine Pedroletti, entretien avec Cédric Lomba, décembre 2006.
} 
Il faut revenir ici sur la formule «contacts négatifs» employée par le journaliste du Nouvel Observateur d'alors. Si l'expression ne renvoie pas à des premières rencontres, elle correspond néanmoins à une réalité. Les initiatives entre étudiants et ouvriers accentuent une prévention croisée, PC-CGT vs UNEF-gauchistes-CFDT. La chose n'est pas tout à fait nouvelle sur le plan national : le film La Chinoise de Godard ou la chanson Pauvres petits c... de Jean Ferrat, qui datent de 1967, l'illustrent bien. Elle prend une force nouvelle en maijuin, des grilles fermées de Renault-Billancourt aux articles publiés par L'Humanité contre les «faux révolutionnaires ». Localement, deux écrits du même auteur donnent la teneur de la discordance : ils sont l'œuvre de Pol Cèbe, lui-même bachelier, ancien soldat en Indochine puis ouvrier à la Rhodia. Cégétiste de choc devenu membre du Parti Communiste en $1966^{13}$, il milite de longue date pour la diffusion de la culture en milieu ouvrier. Il est co-fondateur, avec des enseignants militants chrétiens et un menuisier cédétiste, d'un centre culturel de quartier très actif à Besançon, le Centre culturel de Palente près les Orchamps (CCPPO), inspiré du réseau Peuple et Culture, qui invite des artistes et a suscité la venue de Chris Marker en 1967. Il est par ailleurs responsable de la bibliothèque du Comité d'entreprise de Rhodia, marquante pour bien des ouvriers. De ses relations difficiles avec les étudiants, voici un premier souvenir, évoquant une intervention en $\mathrm{AG}$ à l'usine : "En voulant expliquer, bien timidement, que je connaissais comme ça des gens, et même des professeurs, et même des étudiants, qui n'avaient pas attendu mai 68 pour découvrir que le patrimoine culturel appartient aussi au prolétariat, et même tiens, j'y pense, j'en connaissais des qui depuis des années travaillaient avec les organisations ouvrières d'ouvriers, comme ça, tout doucement, sans bruit, sans barricades, avec des ptits appareils de projection, des ptits bouquins (...). Mais moi, je suis communiste! Alors, un communiste s'adressant, en mai 68, à Rhodiaceta, à une assemblée mi-étudiante mi-CFDT, tu parles $! . .{ }^{14}{ }^{»}$. Le même militant est responsable du journal CGT de la Rhodia, qui donne une appréciation des prises de parole effectuées dans l'usine, le 21 mai 68. Si, selon ce bulletin, les représentants syndicaux enseignants (SNESup, SGEN) expriment un réel programme, les quelques étudiants intervenus « $n$ 'exposent rien sinon leurs complexes petit-bourgeois, découvrant, 50 ans après leur faillite, les vertus des Université populaires». L'article explique ensuite que «la funeste aventure dans

\footnotetext{
${ }^{13}$ Dictionnaire biographique. Mouvement ouvrier, mouvement social (1940-1968), Tome 3.Paris, L'Atelier, 2007.

${ }^{14}$ Pol Cèbe, Livre d'or de la grève de Rhodiaceta, Besançon, Musée du Temps, 1968.
} 
laquelle gaullistes et gauchistes ont failli entraîner la classe ouvrière, n'a été déjouée que grâce à la clairvoyance de la CGT et à l'attitude ferme de ses militants à tous les échelons ${ }^{15} »$. Intellectuels, professeurs et artistes sollicités ès qualités aux côtés du parti de la classe ouvrière ou de la CGT contre étudiants faisant irruption à l'extrême gauche du champ politique, la distinction s'aiguise aux yeux d'une partie des acteurs de mai-juin 1968. Elle se perpétuera en partie après la reprise. Les échanges, en ce sens, ont bien une dimension négative. Mais les relations entre étudiants et ouvriers ne peuvent se lire au seul niveau des organisations et des groupes constitués.

\section{Le moment 68, à replacer dans une temporalité moyenne}

Il faut lire avec prudence les rencontres entre groupes sociaux, notamment en termes de destins générationnels. À l'échelle des personnes et de leurs parcours, il est toutefois possible d'observer des inclinaisons, voire des bifurcations de trajectoires. S'il est admis que les relations des étudiants avec les ouvriers sont liées à leurs trajectoires biographiques (familiales, scolaires et politiques), la perspective symétrique semble moins évidente : sous l'effet, peut-être, d'un regard nostalgique, les ouvriers des années 1960 sont trop souvent appréhendés comme une classe homogène. A y regarder de plus près, les trajectoires sont diverses, parfois zigzagantes. Ces cheminements, en partie lisibles à travers les organisations politiques, s'inscrivent aussi dans des contacts plus directs pris dans les luttes comme on peut le voir à travers deux biographies croisées d'ouvriers leaders des grèves de 67. Georges Maurivard et Nicolas Bultot, nés à la fin des années 30 sont entrés quasiment au même moment à la Rhodia, au début des années 60 , et ont immédiatement rejoint un syndicat pour devenir délégués du personnel. Mais, les luttes de 1967-68 déboucheront pour eux sur des conversions en sens inverse : le premier passe de la CFDT à la CGT-PC en 67 et le second de la CGT-PC à la CFDT-PSU en 68. Pour comprendre ces virages, il est nécessaire d'appréhender, avec d'autres dimensions (familiale, scolaire, etc.), leurs rapports aux mondes intellectuels.

G. Maurivard, pupille de la nation, issu de milieu catholique, obtient un CAP de modeleur sur bois. Il devient délégué du personnel CFDT en 64 et presqu'immédiatement adhère au CCPPO. Il y rencontre les milieux culturels et se lie d'amitié avec Pol Cèbe et

\footnotetext{
${ }^{15}$ Union Rhodia, $\mathrm{n}^{\circ}$, Juin-Juillet 68
} 
d'autres communistes. Discutant facilement avec des étudiants trotskystes en 67, il adhère au PC pour suivre la figure de P. Cèbe. En 68, il maintient les contacts avec les étudiants mais moins intensément qu'en 67 et rejoint la CGT.

N. Bultot est fils unique d'une réfugiée espagnole militante du Parti socialiste espagnol et d'un père brigadiste. Doté d'un simple certificat d'études, il passe par divers petits métiers ouvriers à partir de 13 ans. Il prend des responsabilités syndicales à la Rhodia en 63. Tenant d'une ligne de confrontation dure, considérant que les cégétistes proches du CCPPO sont des « intellectuels », il dialogue avec des étudiants gauchistes durant les conflits, se rend régulièrement à la fac en mai-juin 1968, discute avec des étudiants de lettres et de sciences et poursuit les grèves après Grenelle. Lâché par le PC, et sous le coup d'une menace d'extradition, il quitte la CGT pour la CFDT en 68.

Ces deux parcours sont affectés par les liens avec les mondes intellectuels et de manière durable. G. Maurivard, ne participe pas, comme d'autres, à l'aventure Medvedkine ${ }^{16}$, mais maintient son engagement syndical (secrétaire de section CGT de Besançon durant les années 70), politique (au PC, adjoint au maire en 1977) et culturel (président des Amis de la Maison du Peuple de Besançon). Partie prenante des combats syndicaux et politiques, N. Bultot est resté en contact avec le monde intellectuel : auprès de Michel Rocard à la direction du PSU, avec des étudiants dans la lutte de LIP en 73, ou dans des formations d'extrême-gauche jusqu'à aujourd'hui. A 47 ans, alors remarié avec une médecin généraliste militante, il obtient un diplôme d'éducateur spécialisé et s'investit dans l'aide aux jeunes en difficulté. Sans démêler ce qui tient d'une tradition militante familiale, des relations avec les syndicalistes ouvriers ou des frottements avec des étudiants ou les milieux culturels, force est de constater qu'en regardant à ce niveau, les échanges entre mondes ouvriers et mondes intellectuels en mai-juin 68 ont marqué durablement des parcours à des degrés divers.

\footnotetext{
${ }^{16}$ Bruno Muel, Françine Muel-Dreyfus, « Week-end à Sochaux (1968-1974) », in D. Damamme et alii (dir.), op. cit., p. 329-343.
} 